\title{
COMPARISON OF IMAGE ANALYSIS METHODS ON THE EXAMPLE OF ULTRASONIC THERMOGRAPHY OF AN ARAMID COMPOSITE
}

\author{
Waldemar Świderski \\ Military Institute of Armament Technology \\ Wyszyńskiego Street 7,05-220 Zielonka, Poland \\ tel.: +48227614 552,fax: +48227614677 \\ e-mail:walemar.swiderski@wp.pl
}

\begin{abstract}
Infrared thermography using ultrasound thermal excitation of the tested material is one of the most effective methods in non-destructive testing of a multi-layer aramid composite. This type of material is very popular in the construction of light ballistic armours. Typical defects are delamination between layers of aramid fabric joined by resin. They are usually filled with air. Delamination located deep under the surface of the test generates very weak temperature signals. They are often at the level of noise. To reduce the impact of noise on the detection of a defect, special methods of image analysis (thermograms) are used. Such methods include principal component analysis and wavelet analysis. Principal Component Analysis is a relatively new procedure of statistical data treatment, which is becoming increasingly popular in non-destructive testing. Mathematically, it is often regarded as implementation of the so-called singular values decomposition technique, which allows extracting of spatial information from a matrix of source data. The wavelet analysis is an integral transform, which represents the convolution of an analysed process with a special mother function called wavelet. Wavelets are characterized by two parameters: scale and shift. The paper presents a comparison of the efficacy of these methods in the detection of defects in the multilayer composite reinforced aramid fibre.
\end{abstract}

Keywords: non-destructive testing, composite material, IR thermography, image analysis

\section{Introduction}

Composite materials have recently been used frequently in lightweight ballistic armour designs because advances in polymer chemistry have enabled the production of materials to protect against impacts from small arms and fragments. Woven (textile) materials combined with plastic are usually used, creating multi-layer composite materials suitable for personal ballistic protection (vests and helmets), armoured vehicles, and solid objects. This type of composite material is largely manufactured on the basis of highly resistant aramid and polyethylene fibres combined with phenolic and polyurethane resins and other flexible blends. Such materials are characterized by being lightweight, corrosion-resistant, and easily mouldable, which allows them to be adapted to the surface they are to protect. Material layers can be very different from their physical properties; therefore, such materials are a difficult control task for many traditional non-destructive testing (NDT) techniques. Defects that may appear in this type of multilayer composite material are usually inaccuracies in gluing composite layers and delamination resulting from the impact of fragments and projectiles $[1,2]$.

Active infrared thermography with an ultrasound source of thermal stimulation of the tested material is one of the most effective methods in detecting defects in aramid fibre reinforced composites. Ballistic covers made of this composite have a thickness of up to $10 \mathrm{~mm}$. There is no problem in detecting thin defects in the form of delamination located at a depth of $6-7 \mathrm{~mm}$ below the surface of the tested composite. Problems occur when locating thin defects, which are deeper than $7 \mathrm{~mm}$. The thermal signal generated by damage is often at the level of disturbances generated by the surface of the structure being tested. In these cases, non-standard methods for processing thermograms may be helpful. Such methods include, among others: 
- Fourier analysis,

- differentiating transient signals,

- correlation technique,

- polynomial fitting,

- wavelet analysis,

- Principal Component Analysis.

\section{Image processing}

ThermoFit ${ }^{\mathrm{TM}}$ Pro software developed by prof. V. Vavilova was used for thermogram analysis [3]. This software allows one to use several different algorithms of image processing for the analysis, with the option of changing their parameters.

In order to smooth any high-frequency noise, ThermoFit ${ }^{\mathrm{TM}}$ Pro enables applying a fitting procedure by using three different fitting functions.

The first fitting function (Fit 1 option) follows from the classical solution for heating a semiinfinite adiabatic body with a square pulse:

$$
\begin{aligned}
& T(\tau)=A_{0}+A_{1} \theta+A_{2} \theta^{2}+A_{3} \theta^{3}+A_{4} \theta^{4}+A_{5} \theta^{5} ; \\
& \theta=\sqrt{\frac{\tau}{\tau_{h}}}-\sqrt{\frac{\tau}{\tau_{h}}-1} ; \quad \tau \geq \tau_{h},
\end{aligned}
$$

where $\tau_{h}$ is the heating time.

The second fitting function (Fit 2 option) is general and independent of the physics of the analysed process:

$$
T(\tau)=A_{0}+A_{1} \tau+A_{2} \tau^{2}+A_{3} \tau^{3}+A_{4} \tau^{4}+A_{5} \tau^{5} .
$$

The third fitting function (Fit 3 option) follows on from the classical solution for heating a semi-infinite adiabatic body with a Dirac (flash) pulse:

$$
\begin{gathered}
\operatorname{Ln}[T(\tau)]=A_{0}+A_{1} \Theta+A_{2} \Theta^{2}+A_{3} \Theta^{3}+A_{4} \Theta^{4}+A_{5} \Theta, \\
\Theta=\operatorname{Ln}[\tau] .
\end{gathered}
$$

Note that the Fit 1 and Fit $\mathbf{2}$ procedures are sensitive to values of all time parameters. Therefore, accurate knowledge of these parameters is advisable for obtaining better results.

Applying the fitting functions above results in calculating of up to six polynomial coefficients are: $A_{0}, A_{1}, A_{2}, A_{3}, A_{4}, A_{5}$. Since these coefficients can be calculated for each image pixel $(i, j)$, the Program allows producing synthetic images of polynomial coefficients: $A_{0}, A_{1}, A_{2}, A_{3}, A_{4}, A_{5}$.

The Fourier transform is applied to a whole image sequence ( $\mathrm{N}$ images in a sequence) except the Image No. 1 . If $T(i, j, k)$ is the temporal evolution in the pixel $[i, j]$, where $k=2-N$, the Program allows producing the image of magnitude and phase according to:

$$
\begin{aligned}
& \text { Magnitude }=\sqrt{[\operatorname{Re}(T)]^{2}+[\operatorname{Im}(T)]} \\
& \text { Phase }=\text { ArcTan }\left[\frac{\operatorname{Re}(T)}{\operatorname{Im}(T)}\right],
\end{aligned}
$$

where:

$\operatorname{Re}(T)$ and $\operatorname{Im}(T)$ represents the real and imaginary part of the Fourier transform applied to the $T(i, j, k)$ function. 
The Derivative option allows differentiating pixel-based values through the sequence. The differentiation formula is as follows:

$$
\text { Dif }(i, j, k)=T(i, j, k+1)-T(i, j, k)
$$

where $k=2-N-1 ; N-$ the number of images in the current sequence. Note that the Image No. 1 (ambient temperature image) is omitted in computing derivatives.

Analysing derivatives is a standard technique used to reveal the peculiarities hidden in signal temporal evolutions. For example, a maximum in the first derivative corresponds to the maximum growth rate of the analysed function that can be used in distinguishing between sound and defect areas.

The correlation technique is intended for revealing similarity in the temporal behaviour of the temperature between a chosen reference point and each image pixel. A reference point is chosen by the use then, the correlation image will show pixel-based values of the correlation coefficient $r(i, j)$ according to the following formula:

$$
r(i, j)=\frac{\sum_{k=1}^{N} T(i, j, k) T\left(i_{r e f}, j_{r e f}, k\right)-\frac{1}{N} \sum_{k=1}^{N} T(i, j, k) \bullet \sum_{k=1}^{N} T\left(i_{r e f}, j_{r e f}, k\right)}{\sqrt{\left\{\sum_{k=1}^{N} T^{2}(i, j, k)-\frac{1}{N}\left[\sum_{k=1}^{N} T(i, j, k)\right]^{2}\right\} \bullet\left\{\sum_{k=1}^{N} T^{2}\left(i_{r e f}, j_{r e f}, k\right)-\frac{1}{N}\left[\sum_{k=1}^{N} T\left(i_{r e f}, j_{r e f}, k\right)\right]^{2}\right\}}}
$$

where $T(i, j, k)$ the pixel value in the $\mathrm{k}$-th image is, $T\left(i_{r e f}, j_{r e f}, k\right)$ is the pixel value in a reference point, and $N$ is the number of images in the sequences except the first one.

Principal Component Analysis (PCA) is a relatively new procedure of statistical data treatment, which is becoming increasingly popular in non-destructive testing [4]. Mathematically, it is often regarded as implementation of the so-called singular values decomposition technique, which allows extracting of spatial/temporal information from a matrix of source data.

The PCA method is intended for extracting both spatial and temporal peculiarities from the IR thermographic sequence of source data (source matrix). A 3D matrix is first converted into a 2D matrix; this 2D matrix is then decomposed and the resulting matrix can be represented as a sequence of principal component images.

One of the most important advantages of this technique is that it can be applied to any image sequence without knowledge of its physics and without any data pre-processing.

Wavelet analysis, which underlines frequency composition of time-dependent functions, is a powerful alternative to the Fourier transform. The disadvantage of the Fourier transform is that Fourier frequency components cannot be localized in time, thus limiting the use of the Fourier transform in the analysis of transient processes [5]. In other words, the Fourier transform is unable to show the difference between identical signals which occur at different times within a time of interest.

The wavelet analysis is an integral transform, which represents the convolution of an analysed process with a special mother function called wavelet. Wavelets are characterized by two parameters: scale and shift. The scale is responsible for frequency composition of signals while the shift shows where specific frequencies occur within the period of interest.

\section{Experimental testing}

Samples with dimensions $(100 \times 100) \mathrm{mm}$ contained introduced defects in the form of air gaps, each with dimensions $(10 \times 10) \mathrm{mm}$ and a thickness of approx. $0.1 \mathrm{~mm}$. The defects were located between the $1^{\text {st }}$ and $5^{\text {th }}$ layer of aramid (defect D1), between the $8^{\text {th }}$ and $12^{\text {th }}$ layer of aramid (defect D2), and between the $16^{\text {th }}$ and $20^{\text {th }}$ layer of aramid (defect D3).

Ultrasonic stimulation was carried out by means of an ultrasonic generator with a frequency of 
$25 \mathrm{kHz}$. The output power was $300 \mathrm{~W}$ (maximum allowable power was $2 \mathrm{~kW}$ ). The time of generating of the ultrasound signal was $1 \mathrm{~s}$.

In Fig. 1., a test stand with a sample of the tested material is presented. In Fig. 2., a thermogram is shown where the deepest located defect, D3, is very poorly visible.

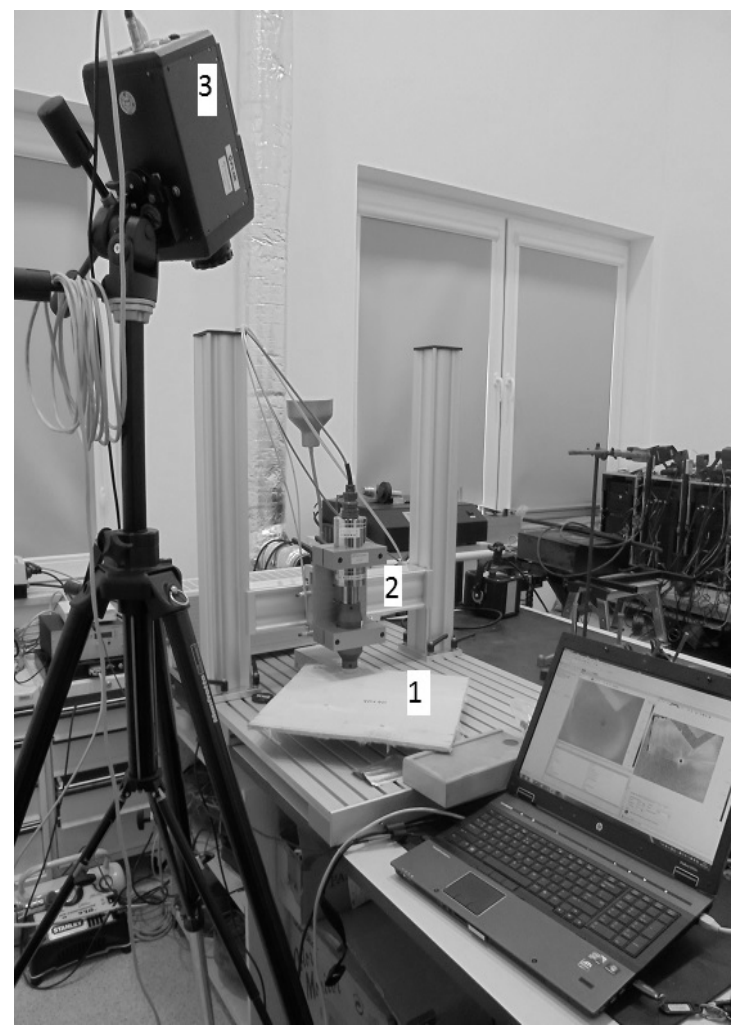

Fig. 1. The test stand: 1 -sample, 2 -ultrasonic source, 3 -thermal camera

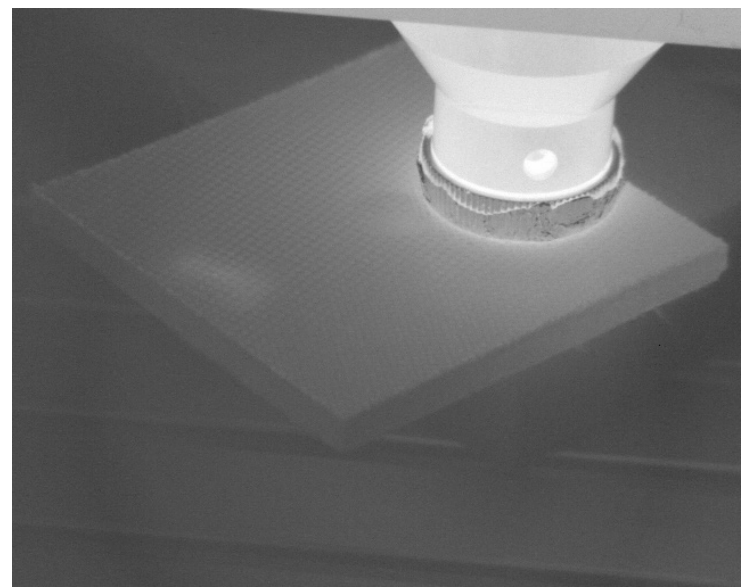

Fig. 2. A sample of a multi-layer aramid composite with an air defect D3-the best thermogram of the sequence

Over the defect D1, closest to the surface of the tested composite, the maximum temperature increase was at 0.73 to $1.21^{\circ} \mathrm{C}$ at different frequencies of generated ultrasound. For the defect D2, located in the middle of the thickness of the structure under investigation, the maximum temperature increase ranged from 0.33 to $0.84^{\circ} \mathrm{C}$. For the $\mathrm{D} 3$ defect, located deepest below the surface, the increment was the lowest, from 0.17 to $0.28^{\circ} \mathrm{C}$.

The following algorithms were used to improve the illustration of the D3 defect presented on the thermogram (Fig. 2): Fourier transforms, ply nominal fitting, derivative analysing, PCA and wavelet analysis. The obtained views are presented in Fig. 3-5. 


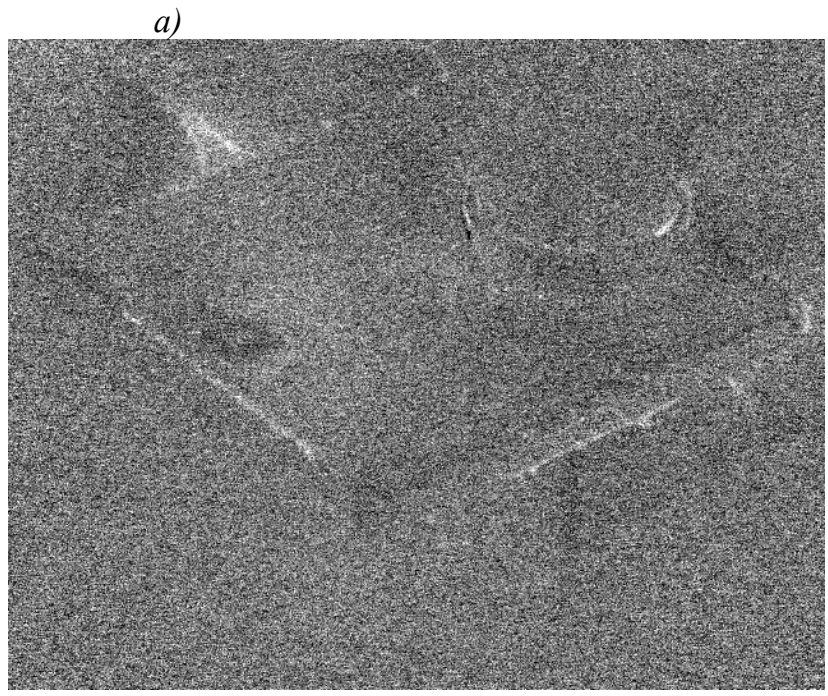

b)

Fig. 3. Thermograms of a sample of a multi-layer aramid composite with an air defect D3 after fitting function: a) $F 2$, b) F3

a)

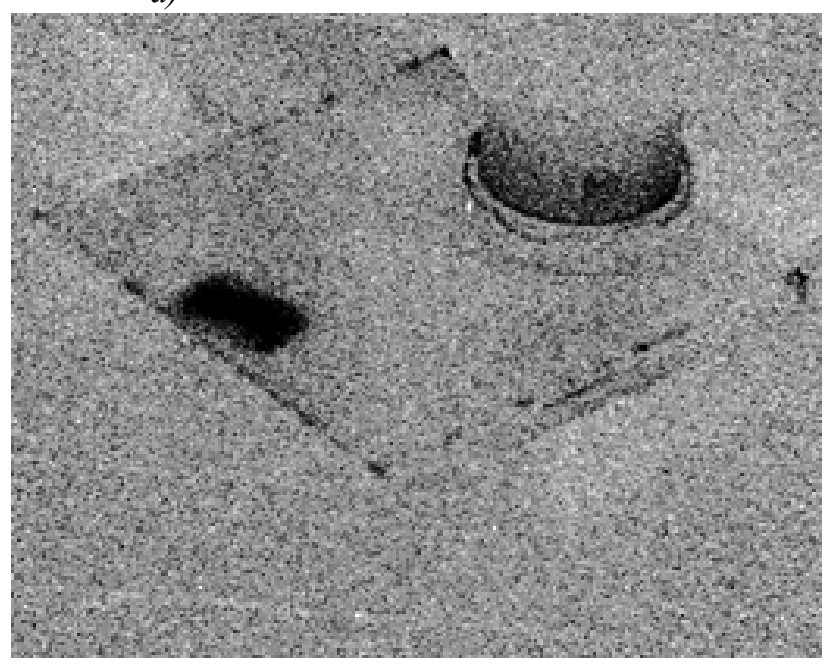

b)

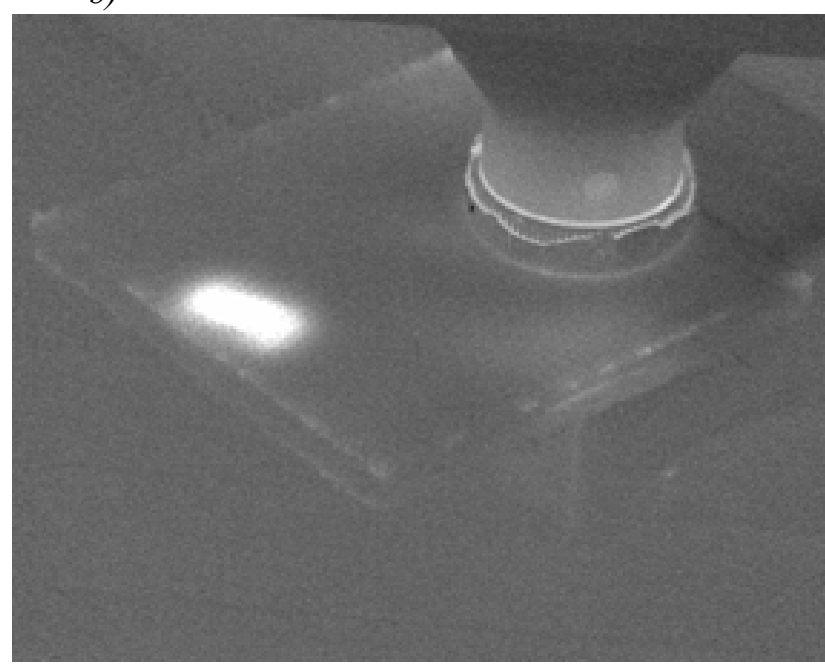

Fig. 4. Thermograms of a sample of a multi-layer aramid composite with an air defect D3 after: a) wavelet analysis - MHT function, b) PCA analysis - showing the second component

\section{Summary}

The comparative processing of data (images) showed that in the case of non-destructive tests of the aramid composite with delamination defect, the best improvement in detection of the defect located deep under the surface of the tested sample was obtained using PCA algorithms and wavelet analysis. The use of wavelet analysis; however, is more complicated. The reason for this is that you can use several different wavelet functions for analysis. In each of these functions, there are several parameters, whose values should be changed to get the best image quality. For this reason, PCA analysis is recommended. In the case of unsatisfactory results from the use of PCA, wavelet analysis should be used.

In this case, it was ineffective to use an algorithm based on analysing derivatives. A slight improvement was obtained using the fitting function. Using a Fourier transform, location of the defect is easy but it is difficult to determine its dimensions because its contours are blurred.

Future work will be directed towards the use of these algorithms in composite materials with a different structure. 


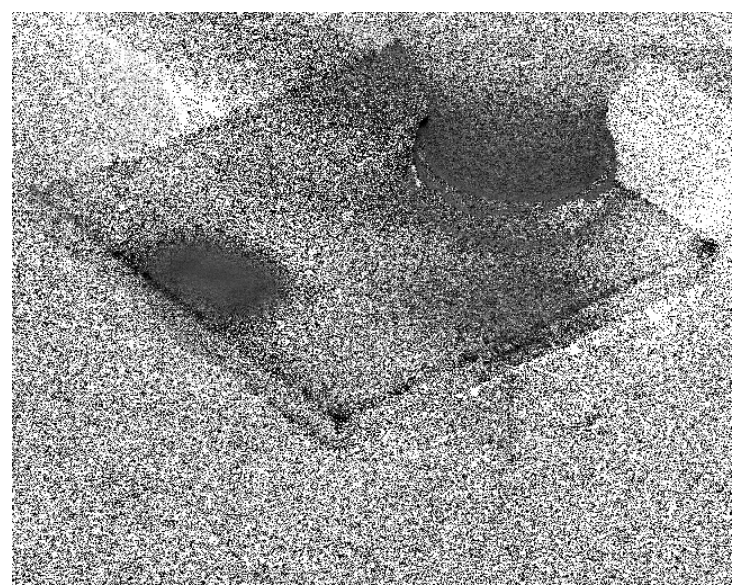

Fig. 5. Thermogram of a sample of a multi-layer aramid composite with an air defect D3 after Fourier transforms

\section{References}

[1] Swiderski, W., Hłosta, P., Non-Destructive Evaluation of Impacted CFRP by IR Thermography, Materials, Vol. 12 (6), 2019.

[2] Swiderski, W., Non-destructive testing of light armours of CFRP after ballistic impacts by IR thermography methods, Composite Structures, Vol. 224, 2019.

[3] ThermoFit Pro Operation Manual, Innovation Ltd., 2016.

[4] Rajic, N., Principal Component Thermography, In Defence Science \& Technology, Airframes and Engines Division Aeronautical and Maritime Research Laboratory: Fairbairn, Australia, Canberra 2002.

[5] Galmiche, F., Vallerand, S., Maldague, X., Wavelet transform applied to pulsed phase thermography, Proceedings of V-th Workshop on Advances in Infrared Technology and Applications, CNR, pp. 117-122, Venice 1999.

Manuscript received 26 March 2019; approved for printing 27 June 2019 